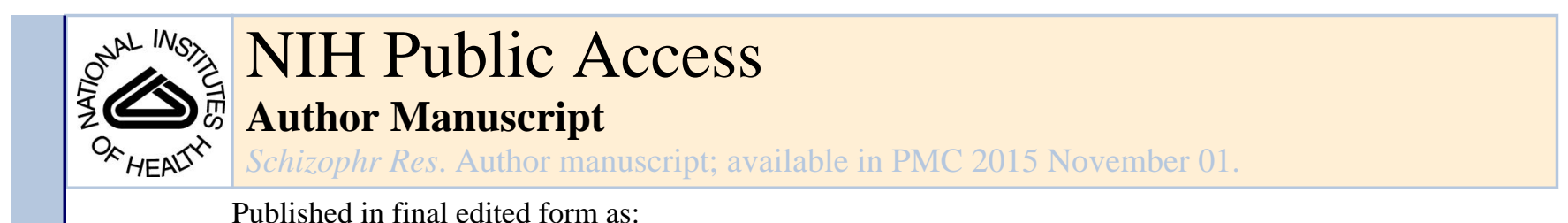

Published in final edited form as:

Schizophr Res. 2014 November ; 159(0): 263-266. doi:10.1016/j.schres.2014.09.031.

\title{
Movement Abnormalities Predict Transitioning to Psychosis in Individuals at Clinical High Risk for Psychosis
}

\author{
Dallas A. Callaway ${ }^{a}$, Diana O. Perkins ${ }^{b}$, Scott W. Woods ${ }^{c}$, Lu Liu ${ }^{a}$, and Jean Addington ${ }^{a}$ \\ Dallas A. Callaway: dacallaw@ucalgary.ca; Diana O. Perkins: diana_perkins@med.unc.edu; Scott W. Woods: \\ scott.woods@yale.edu; Lu Liu: Iuliu@ucalgary.ca; Jean Addington: jmadding@ucalgary.ca \\ aMathison Centre for Mental Health Research \& Education, University of Calgary, 3280 Hospital \\ Drive NW, Calgary, Alberta, T2N 4Z6, Canada \\ ${ }^{b}$ Department of Psychiatry, University of North Carolina, Chapel Hill, North Carolina, USA \\ 'Department of Psychiatry, Yale University, New Haven, Connecticut, USA
}

\section{Abstract}

Improving upon the predictive validity of determining the transition from high risk to actual psychosis is a primary aim of early intervention research. Previous research has suggested that premorbid spontaneous dyskinesias may be one possible predictor. In this study, dyskinetic movements were assessed with the Abnormal Involuntary Movement Scale (AIMS) at baseline of a longitudinal study of 148 individuals at clinical high risk (CHR) of developing psychosis. Twenty-eight individuals transitioned to a psychotic disorder over the course of the study. Group comparisons between transitioned and non-transitioned individuals indicated that, relative to those that were not observed to transition, participants that developed a psychotic disorder exhibited greater spontaneous dyskinesias at baseline. Moreover, increased dyskinetic movements at baseline resulted in a more than two-fold increase in odds of developing a psychosis for each point increase in AIMS scale score. These findings suggest that individuals with greater premorbid dyskinetic movements may comprise a subset of CHR individuals at inordinate risk to decompensate into psychosis. Future work should employ assessments of spontaneous dyskinesias by instrumentation (e.g., electromyography) and look to ascertain whether specific dyskinesias (e.g., dystonia) or dyskinesias of specific body regions are associated with transitioning to psychosis.

(C) 2014 Elsevier B.V. All rights reserved.

Corresponding Author: Dallas A. Callaway, Mathison Centre for Mental Health Research \& Education, University of Calgary, 1D63 TRW Building, 3280 Hospital Drive NW, Calgary, Alberta, T2N 4Z6, Canada. Phone: 1-403-210-8740. Fax: 1-403-210-9304.

dacallaw@ucalgary.ca.

Contributors

Drs. Addington, Perkins and Woods were responsible for the design of the study and the supervision of all aspects of data collection. Lu Liu and Dallas Callaway were responsible for data analysis. Dallas Callaway took the lead on writing the manuscript with help in writing from Dr. Addington. All authors contributed to and approved the final manuscript.

Conflict of interest

There are no conflicts of interest for any of the authors with respect to the data in this paper or for the study.

Publisher's Disclaimer: This is a PDF file of an unedited manuscript that has been accepted for publication. As a service to our customers we are providing this early version of the manuscript. The manuscript will undergo copyediting, typesetting, and review of the resulting proof before it is published in its final citable form. Please note that during the production process errors may be discovered which could affect the content, and all legal disclaimers that apply to the journal pertain. 


\section{Keywords}

psychosis; schizophrenia; dyskinesia; movement abnormality; conversion; prodromal

\section{Introduction}

Within the high risk literature, there is a major focus on identifying predictors of the transition to psychosis. Current estimates specify that, amongst individuals meeting formal operationalized criteria (e.g., Yung et al., 2005; McGlashan et al., 2010) for being at clinical high risk to develop psychosis (CHR), 36\% will go on to develop a full-blown psychotic disorder within three years (Fusar-Poli et al., 2012). As part of this effort, premorbid movement abnormalities - namely hyperkinetic movements such as non-drug induced choreoathetoid and ballistic, dystonic, and stereotypic movements - which are collectively referred to as spontaneous dyskinesias have been suggested as potential predictors of psychotic transition. This is largely due to the presence of these behaviors among youth displaying other signs of psychosis-risk (MacManus et al., 2012), the prevalence of these symptoms among CHR individuals (Mittal et al., 2007), evidence from prospective and follow-back studies of motor abnormalities in those who go on to develop psychosis (Dickson et al., 2012; Rosso et al., 2000; Schiffman et al., 2004), and studies in which medication-free individuals with schizophrenia and other psychotic disorders exhibit dyskinetic movements across stages of illness (Whitty et al., 2009). The overlap between the neural substrate thought to underlie both dyskinesias and psychotic symptoms (Walker, 1994) provides further support for dyskinesias as potential predictors of psychosis onset. In particular, and although not definitive, there is evidence that dopaminergic pathways within the striatum including dopamine neurons projecting from the ventral tegmental area to the nucleus accumbens and caudate nucleus as well as cortico-striato-pallido-thalamic pathways more generally are thought to give rise to both dyskinetic movements and psychotic symptomatology (e.g., Delong \& Wichmann, 2007; Mittal et al., 2008; Walker et al., 1996).

Furthermore, Mittal and Walker (2007) evaluated dyskinetic movements as predictors of transition to psychosis within a CHR sample. This study found that dyskinesias of the face and upper body were associated with increased odds of developing a psychotic disorder. To our knowledge, the work by Mittal and Walker represents the only investigation of premorbid movement abnormalities as predictors of transition to psychosis within a CHR sample. Unfortunately, the prospect of confounding pharmacotherapy (i.e., drug-induced movement disorders) within their sample as well as the small subset of participants who transitioned to psychosis $(n=10)$ limits the extent to which the results of this work may be generalized. The present investigation, therefore, sought to confirm whether premorbid dyskinetic movements predict transition to psychosis using a large, neuroleptic-naïve CHR sample obtained from a multisite longitudinal study.

\section{Method}

\subsection{Participants}

The sample consisted of 148 individuals ( 86 male, 62 female) at CHR for psychosis. All participants were part of a longitudinal NIMH-funded study entitled "Enhancing the 
Prospective Prediction of Psychosis" (PREDICT) that was conducted at the Universities of North Carolina at Chapel Hill, Toronto, and Yale to determine predictors of conversion to psychosis in individuals at CHR. All participants met the Criteria of Prodromal States (COPS) based on the Structured Interview for Psychosis-Risk Syndromes (SIPS; McGlashan et al., 2010). The sample had a mean age of 19.77 (4.60) years and the majority were Caucasian (77\%). One hundred and forty-five (97.98\%) met attenuated positive symptom syndrome (APSS) criteria, one $(0.67 \%)$ met genetic risk and deterioration (GRD) criteria, and two $(1.35 \%)$ met both APSS and GRD criteria. APSS includes the onset or worsening of non-psychotic level disturbances in thought content, thought processes, and/or perceptual abnormalities over the past year whereas GRD requires either a first degree relative with a psychotic disorder diagnosis or the participant having schizotypal personality disorder in conjunction with at least a 30\% drop in functioning on the General Assessment of Functioning (GAF) scale over the past year.

Participants were excluded if they met criteria for any current or lifetime psychotic disorder, had a measured IQ $<70$, had any history of central nervous system disorder or clinically significant head trauma, and if they had any current use of antipsychotic medication at baseline as well as if they received antipsychotic medication at any point during follow-up. Table 1 displays baseline psychotropic medication status for the sample.

\subsection{Measures}

Criteria for a psychosis-risk syndrome and for conversion to psychosis were determined using the SIPS and symptoms were assessed using the Scale of Psychosis-Risk Symptoms (SOPS; McGlashan et al., 2010). The Structured Interview for Axis I DSM-IV Disorders (SCID-I; First et al., 2002) was used to evaluate the presence of any Axis I disorder.

Dyskinetic movements were assessed using the Abnormal Involuntary Movement Scale (AIMS; Guy, 1976). The AIMS is a widely-used 10-item clinician-rated scale that evaluates aberrant movement in three body regions - the orofacial area (items 1-4; e.g., grimacing), extremities (items 5-6; e.g., athetoid movements of the hands), and trunk (item 7; e.g., shoulder rocking) - using a 0 (none/normal) to 4 (severe) scale. The AIMS includes three additional global evaluation items (8-10) that measure global severity of movements, incapacitation due to involuntary movements, and patient awareness of their dyskinesias, respectively. Apart from the individual item scores, the AIMS yields two scale scores: a total score including all 10 items and a non-global score which includes the seven behaviorbased ratings of abnormal movements (items 1-7) and excludes the three global evaluation items (8-10).

\subsection{Procedure}

The SIPS, SCID-I, and AIMS were administered at in-person baseline sessions. Raters were experienced research clinicians who demonstrated adequate reliability at routine SIPS and SCID-I reliability checks. The AIMS was administered by the same research clinicians and were trained to requisite reliability on the measure by review of a training tape and supervision of the study psychiatrist at each site. Post-training inter-rater agreement on determining initial eligibility and subsequent transition status was excellent $(\kappa=0.90)$. A 
clinical psychologist or psychiatrist at each site conducted a comprehensive clinical assessment to determine if entry criteria were met. Inter-rater reliability for the SCID-I was determined at the start of the study and evaluated annually by $100 \%$ agreement on the diagnosis and at least $80 \%$ agreement for symptom presence. J.A. chaired weekly conference calls to review criteria for individuals admitted to the study. The study protocols and informed consents were reviewed and approved by the ethical review boards of all three study sites.

\subsection{Statistical Analyses}

None of the AIMS dependent variables satisfied the normality assumption for parametric statistics and were non-transformable by way of square, square root, and log methods. Moreover, there was a preponderance of zero values within the presented sample. Accordingly, nonparametric Mann-Whitney $U$ tests were used for group comparisons between transitioned and non-transitioned individuals and the $(x+0.5)$ correction was applied to all AIMS variables (Yamamura, 1999). To quantify the predictive power of dyskinetic movements, continuous AIMS total and non-global scores were regressed on dichotomous psychosis transition status (non-transitioned vs. transitioned) in independent logistic regression analyses. Significant multicollinearity among several AIMS item scores precluded interpretation of multiple regression analyses. All analyses were conducted using SPSS (IBM Corp., 2012) and Stata/IC 10.0 software (StataCorp, 2007).

\section{Results}

Of the $148 \mathrm{CHR}$ individuals, $28(18.92 \%)$ made the transition to psychosis. Chi-square, $U$, and $t$ tests indicated that the groups did not significantly differ in age $(p=.86)$, sex $(p=.33)$, ethnicity $(p=.42)$, or educational attainment $(p=.70)$. Further analyses indicated that there were no substantively significant demographic, clinical, or AIMS score difference between participants recruited through the three sites of this study and thus merger of the multisite data was justified.

\subsection{Transition Group Differences in Abnormal Movements}

Table 2 displays AIMS scores for the sample. A series of Mann-Whitney $U$ tests indicated that participants who made the transition to psychosis demonstrated significantly more severe ratings than those who did not make the transition on AIMS total, non-global, severity of abnormal movements, and awareness of movements scores. Those who made the transition also had more severe ratings on specific indices of dyskinetic movements of the facial muscles, jaw, upper extremity, trunk, and, at a trend-level, movements of the tongue.

\subsection{Abnormal Movements and Transition to Psychosis}

Logistic regression analyses indicated that AIMS total $(\beta=0.81, S E=0.28$, Wald $=8.68, p$ $<0.01, \mathrm{OR}=2.25,95 \% \mathrm{CI}=1.31-3.85)$ and non-global $(\beta=0.80, S E=0.32$, Wald $=6.29$, $p=0.01, \mathrm{OR}=2.22,95 \% \mathrm{CI}=1.19-4.15)$ scores were individually significant predictors of transition to psychosis. That is, elevated scores on the AIMS, indicative of greater dyskinetic movements, conferred a greater than two-fold multiplicative increase in odds of developing a psychotic disorder. Table 3 displays the probability of transitioning to psychosis for each 
reported AIMS non-global score in our sample. The non-global score was selected as this index only includes the behavior-based ratings of abnormal movements (items 1-7) and not the global evaluation items (8-10) and is thus considered a relatively pure measure of dyskinetic movements.

\section{Discussion}

This multisite, longitudinal study investigated whether premorbid dyskinetic movements predict transitioning to psychosis among CHR individuals. The presence of dyskinetic movements within the presented neuroleptic-naïve CHR sample suggest that, although dyskinesias are well-documented pharmacologic sequelae, these movements may reflect a primary, rather than secondary, component of the psychosis disease process. This is consistent with multiple investigations of pre-psychosis individuals (Rosso et al., 2000; Walker et al., 1994) and psychotic disorder patients (Whitty et al., 2009) in which movement abnormalities were observed in the absence of antipsychotic medication. Viewed in this manner, dyskinetic movements may not only constitute potential predictors of psychosis onset but also comprise a set of symptoms that predate the onset of psychotic symptomatology and endure through the course of illness episodes thereby warranting independent clinical attention.

The observation of elevated baseline movement abnormalities for those that converted to psychosis relative to those that did not, in conjunction with the result that elevated dyskinetic movements induced a more than doubling of odds to develop a psychosis for each point increase in AIMS scores suggest that premorbid movement abnormalities may be used to enhance the prediction of psychosis onset. These findings closely mirror those of prior work in which dyskinetic movements singly (Mittal \& Walker, 2007) or jointly with other risk factors (e.g., neurocognitive deficits; Mittal et al., 2010) have been shown to predict the onset of psychosis.

Interestingly, the presented pattern of results also fit with prior work documenting the predictive power of spontaneous dyskinesias within specific body regions. For example, across several investigations including samples of schizophrenia (Puri et al., 1999) and schizophrenia-spectrum disorder (Mittal et al., 2007; Walker et al., 1999) patients as well as CHR individuals (Mittal \& Walker, 2007), dyskinetic movements in the facial and upperbody regions have been shown to occur with greater frequency than dyskinesias of the lower-body (cf. Mittal et al., 2008). Similarly, our results indicated greater dyskinesias in the muscles of facial expression, jaw, tongue, upper extremity, and trunk within CHR individuals who transitioned to psychosis relative to those who did not. These findings should be viewed with caution as multicollinearity barred analysis of facial and upper-body region AIMS items as independent predictors of psychosis and correcting for Type I error across the family of group contrasts would exclude differences in dyskinesias of the tongue and facial muscles from conventional levels of statistical significance. Nonetheless, given that abnormal movements of the upper body and face, but not lower body, delineated at baseline those who do and do not transition to psychosis in this and at least one other study (Mittal \& Walker, 2007), it is notable that the movement abnormalities that predate transitioning to psychosis may not be generalized motor problems but rather more 
circumscribed profiles of facial and upper body motor dysregulation. These may reflect distinct underlying neuropathologies that match the topographical organization of the motor cortex and associated midbrain structures and thus point to specific neurocircuitries possibly implicated in the etiology and pathophysiology of psychosis (Lewis \& Levitt, 2002; Walker, 1994). Clearly, however, as these postulations remain highly speculative, further work is needed to elucidate the predictive validity of specific dyskinetic movements or dyskinesias in specific body regions with regards to psychosis onset and whether these behaviors share etiologic origins with psychotic symptomatology.

There are several limitations worth noting. First, our assessment of spontaneous dyskinesias was based upon a clinician-rated instrument. Although the AIMS is a widely used measure with adequate to good psychometric properties (Guy, 1976), there are notable drawbacks to using rating scales to assess movement abnormalities such as insensitivity of these measures to minor movement problems (Dean et al., 2004) and difficulties demarcating voluntary and involuntary dyskinetic movements. Accordingly, future studies should look to confirm the present findings using biotechnological devices (e.g., force gauges, position transducers, accelerometers, electromyography) to assess dyskinetic movements. As well, future studies should document any indications from participants clinical history or presentation (e.g., abrupt onset, inconsistent presence) that may serve to disambiguate whether the displayed movements have any volitional basis (Fahn, 2012). Second, although raters were trained to a high standard initially, ongoing assessment of reliability was not conducted in this study. Third, as shown in Table 3, only a minority of CHR individuals $(n=7)$ that transitioned to psychosis exhibited a non-zero AIMS non-global score. Although a greater proportion of transitioning (25\%) than non-transitioning (8.33\%) CHR individuals exhibited a non-zero AIMS non-global score, the statistical power of this sample is overtly limited and further work is clearly needed to provide more reliable and externally valid estimates of the increased odds of developing a psychosis given greater abnormal movements. Lastly, as is typical of longitudinal research, some CHR individuals discontinued participation in the study during follow-up and their omission may therefore bias the presented results.

In conclusion, the results of this study support a previous and smaller investigation positing premorbid dyskinetic movements as predictors of transition to psychosis among CHR individuals. This is significant in that individuals with spontaneous dyskinesias may represent a subset of the CHR population that is at particular risk to develop psychosis.

\section{Acknowledgments}

\section{Role of funding source}

Funding for this study was provided by NIMH grants U01MH06634-02 to Dr. Addington, U01MH066069-04 to Dr. Perkins, and U01MH066160 to Dr. Woods. The National Institutes of Mental Health had no further role in study design; in the collection, analysis, and interpretation of data; in the writing of the report; or in the decision to submit the paper for publication.

This study was supported by the following NIMH grants U01MH06634 to J. Addington, U01MH066069 to D. Perkins, U01MH066160 to S. Woods. 


\section{References}

Dean CE, Russell JM, Kuskowski MA, Caligiuri MP, Nugent SM. Clinical rating scales and instruments: how do they compare in assessing abnormal, involuntary movements? J Clin Psychopharmacol. 2004; 24:298-304. [PubMed: 15118484]

DeLong MR, Wichmann T. Circuits and circuit disorders of the basal ganglia. Arch Neurol. 2007; 64:20-24. [PubMed: 17210805]

Dickson H, Laurens KR, Cullen AE, Hodgins S. Meta-analyses of cognitive and motor function in youth aged 16 years and younger who subsequently develop schizophrenia. Psychol Med. 2012; 42:743-755. [PubMed: 21896236]

Fahn, S. Psychogenic movement disorders. In: Albanese, A.; Jankovic, J., editors. Hyperkinetic Movement Disorders: Differential Diagnosis and Treatment. Blackwell; New York: 2012. p. 375-384.

First, M.; Spitzer, RL.; Gibbon, M.; Williams, B.; Williams, JBW. Structured Clinical Interview for DSM-IV Axis I Disorders, Patient Edition. Biometrics Research Department, New York State Psychiatric Institute; New York, New York: 1995.

Fusar-Poli P, Bonoldi I, Yung AR, Borgwardt S, Kempton MJ, Valmaggia L, Barale F, Caverzasi E, McGuire P. Predicting psychosis: meta-analysis of transition outcomes in individuals at high clinical risk. Arch Gen Psychiatry. 2012; 69:220-229. [PubMed: 22393215]

Guy, W. ECDEU Assessment Manual for Psychopharmacology. US Department of Health, Education, and Welfare, District of Columbia; Washington: 1976.

IBM Corp. IBM SPSS Statistics for Windows, Version 21.0. IBM Corp; Armonk: 2012.

Lewis DA, Levitt P. Schizophrenia as a disorder of neurodevelopment. Annu Rev Neurosci. 2002; 25:409-432. [PubMed: 12052915]

MacManus D, Laurens KR, Walker EF, Brasfield JL, Riaz M, Hidgins S. Movement abnormalities and psychotic-like experiences in childhood: markers of developing schizophrenia? Psychol Med. 2012; 42:99-109. [PubMed: 21740623]

McGlashan, T.; Walsh, B.; Woods, S. The Psychosis-Risk Syndrome: Handbook for Diagnosis and Follow-Up. Oxford University Press; New York: 2010.

Mittal VA, Dhruv S, Tessner KD, Walder DJ, Walker EF. The relations among putative biorisk markers in schizotypal adolescents: minor physical anomalies, movement abnormalities, and salivary cortisol. Biol Psychiatry. 2007; 61:1179-1186. [PubMed: 17188254]

Mittal VA, Neumann C, Saczawa M, Walker EF. Longitudinal progression of movement abnormalities in relation to psychotic symptoms in adolescents at high risk of schizophrenia. Arch Gen Psychiatry. 2008; 65:165-171. [PubMed: 18250254]

Mittal VA, Walker EF. Movement abnormalities predict conversion to Axis I psychosis among prodromal adolescents. J Abnorm Psychol. 2007; 116:796-803. [PubMed: 18020725]

Mittal VA, Walker EF, Bearden CE, Walder D, Trottman H, Daley M, Simone A, Cannon TD. Markers of basal ganglia dysfunction and conversion to psychosis: neurocognitive deficits and dyskinesias in the prodromal period. Biol Psychiatry. 2010; 68:93-99. [PubMed: 20227679]

Puri BK, Barnes TRE, Chapman MJ, Hutton SB, Joyce EM. Spontaneous dyskinesia in first episode schizophrenia. J Neurol Neurosurg Psychiatry. 1999; 66:76-78. [PubMed: 9886457]

Rosso IM, Bearden CE, Megginson J, Gasperoni TL, Sanchez LE, Hadley T, Cannon TD. Childhood neuromotor dysfunction in schizophrenia patients and their unaffected siblings: A prospective cohort study. Schizophr Bull. 2000; 26:367-378. [PubMed: 10885637]

Schiffman J, Walker E, Ekstrom M, Schulsinger F, Sorensen H, Mednick S. Childhood videotaped social and neuromotor precursors of schizophrenia: a prospective investigation. Am J Psychiatry. 2004; 161:2021-2027. [PubMed: 15514402]

StataCorp. Stata Statistical Software: Release 10. StataCorp LP: College Station; 2007.

Walker E, Lewis N, Loewy R, Palyo S. Motor dysfunction and risk for schizophrenia. Dev Psychopathol. 1999; 11:509-523. [PubMed: 10532622]

Walker EF. Developmentally moderated expressions of the neuropathology underlying schizophrenia. Schizophr Bull. 1994; 20:453-480. [PubMed: 7526447] 
Walker EF, Lewine RRJ, Neumann C. Childhood behavioral characteristics and adult brain morphology in schizophrenia. Schizophr Res. 1996; 22:93-101. [PubMed: 8958592]

Walker EF, Savoie T, Davis D. Neuromotor precursors of schizophrenia. Schizophr Bull. 1994; 20:441-451. [PubMed: 7526446]

Whitty PF, Owoeye O, Waddington JL. Neurological signs and involuntary movements in schizophrenia: Intrinsic to and informative on systems pathobiology. Schizophr Bull. 2009; 35:415-424. [PubMed: 18791074]

Yamamura K. Transformation using $(\mathrm{x}+0.5)$ to stabilize the variance of populations. Res Popul Ecol. 1999; 41:229-234.

Yung AR, Yuen HP, McGorry PD, Phillips LJ, Kelly D, Dell Olio M, Francey SM, Cosgrave EM, Killackey E, Stanford C, Godfrey K, Buckby J. Mapping the onset of psychosis: the Comprehensive Assessment of At-Risk Mental States. Aust N Z J Psychiatry. 2005; 39:964-971. [PubMed: 16343296] 


\section{Table 1}

Psychotropic medications at baseline.

\begin{tabular}{lcc}
\hline Medication & \multicolumn{2}{c}{ Frequency $(\boldsymbol{\%})^{\boldsymbol{a}}$} \\
\hline None & Transition to psychosis $(\boldsymbol{n}=\mathbf{2 8})$ & Non-transition to psychosis $(\boldsymbol{n}=\mathbf{1 2 0})$ \\
Sertraline & $22(78.57 \%)$ & $74(61.67 \%)$ \\
Fluoxetine & $3(10.71 \%)$ & $9(7.50 \%)$ \\
Citalopram & $1(3.57 \%)$ & $5(4.17 \%)$ \\
Escitalopram Oxalate & $1(3.57 \%)$ & $4(3.33 \%)$ \\
Paroxetine & $1(3.57 \%)$ & $3(2.50 \%)$ \\
Fluvoxamine & 0 & $1(0.83 \%)$ \\
Trazodone & 0 & $1(0.83 \%)$ \\
Venlafaxine & $1(3.57 \%)$ & 0 \\
Mirtazapine & 0 & $2(1.67 \%)$ \\
Bupropion & 0 & $2(1.67 \%)$ \\
Clonazepam & 0 & $8(6.67 \%)$ \\
Lorazepam & $1(3.57 \%)$ & $4(3.33 \%)$ \\
Alprazolam & 0 & $2(1.67 \%)$ \\
Methylphenidate & 0 & $1(0.83 \%)$ \\
Phentermine & 0 & $2(1.67 \%)$ \\
Atomoxetine & 0 & $1(0.83 \%)$ \\
\hline & 0 & $1(0.83 \%)$ \\
\hline
\end{tabular}

${ }^{a}$ Reported frequencies do not distinguish monotherapy and polypharmacy treatments (e.g., one CHR individual that transitioned to psychosis received combined trazodone, sertraline, and clonazepam treatment). 


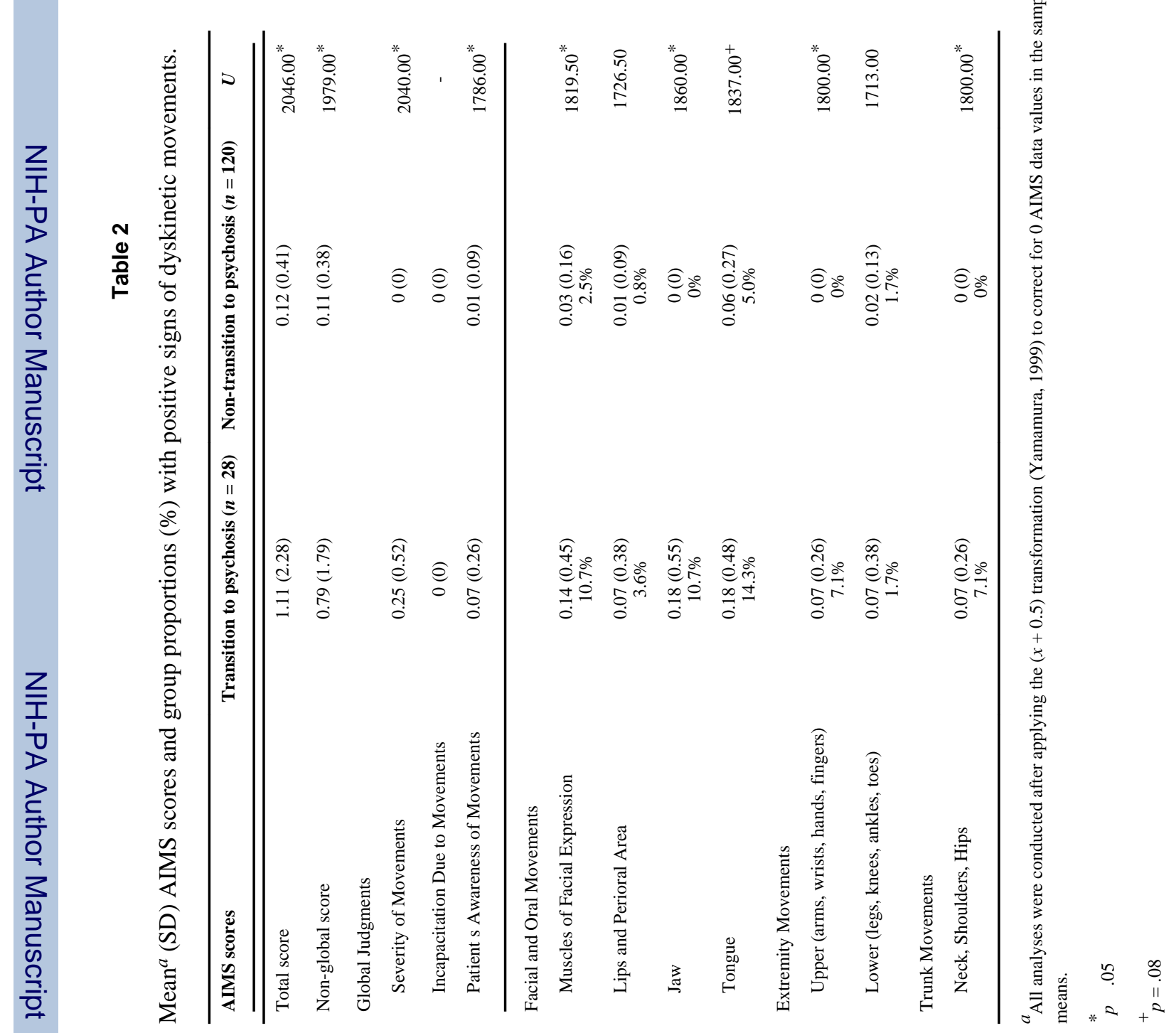


Table 3

Risk of transitioning to psychosis associated with respective AIMS non-global scores.

\begin{tabular}{ccccc}
\hline AIMS Non-Global Score & Full Sample $\boldsymbol{n}$ & Transition Risk $^{\boldsymbol{a}}$ & $\mathbf{9 5 \%}$ CI & Transitioned to Psychosis $\boldsymbol{n}$ \\
\hline 0 & 131 & 0.16 & $0.10-0.23$ & 21 \\
1 & 9 & 0.29 & $0.18-0.44$ & 2 \\
2 & 5 & 0.48 & $0.22-0.75$ & 2 \\
3 & 1 & 0.67 & $0.26-0.92$ & 1 \\
6 & 1 & 0.96 & $0.38-0.99$ & 1 \\
7 & 1 & 0.98 & $0.42-0.99$ & 1 \\
\hline
\end{tabular}

${ }^{a}$ Probability of transitioning to psychosis given specified AIMS non-global score. 